\title{
Diseño de estrategias para mejorar la imagen de hoteles: caso hotel Brisas Trinidad del Mar (Cuba)
}

\author{
Dayana Duffus Miranda* \\ Universidad Central "Marta Abreu" de Las Villas (Cuba) \\ Roberto Carmelo Pons García** \\ Universidad de Matanzas (Cuba)
}

\begin{abstract}
Resumen: Esta investigación se enfoca a evaluar la imagen de hoteles en el proceso de dirección estratégica, saldando los vacíos teóricos y metodológicos relacionados con la gestión de este recurso intangible. La misma aporta un modelo conceptual que explica cómo la imagen de un hotel está determinada por factores subjetivos y objetivos. Otro resultado de esta investigación es el procedimiento que se propone, donde los instrumentos de medida que lo componen se aplican a 1395 encuestados y se somete al criterio de expertos para comprobar científicamente que con la evaluación de la imagen en la dirección estratégica de hoteles en Cuba es posible lograr su imagen deseada. En este estudio se expone además, de forma íntegra, la aplicación del procedimiento propuesto en el hotel: Brisas Trinidad del Mar.
\end{abstract}

Palabras Clave: Imagen deseada; Hoteles; Dirección estratégica.

Design strategies to improve the image of hoteles: hotel case Brisas Trinidad del Mar (Cuba)

Abstract: This research focuses on evaluating the image of hotels within the strategic management process, resolving the theoretical and methodological gaps relating to the treatment of this intangible resource. It provides a conceptual model that explains how the image of a hotel is determined by subjective and objective factors. It also proposes a procedure already experimented with in Cuba where the evaluatory measures were submitted to a total cohort of 1,395 respondents and subjected to expert analysis to verify scientifically that it is possible to achieve the desired image projection through strategic control within the hotel management process in Cuba.. The case used as an example was the Hotel Brisas Trinidad del Mar.

Keywords: Desired image; Hotels; Strategic management.

\section{Introducción}

El turismo se ha convertido en uno de los actores trascendentales en el comercio internacional y al mismo tiempo representa una de las principales fuentes de ingreso de numerosos países en desarrollo. Para el caso específico de Cuba este sector constituye la sexta actividad económica en aportes al Producto Interno Bruto (PIB), y representa un sector clave para la especialización productiva (ONEI, 2015). A pesar del peso determinante de este sector para la economía cubana, el mismo presenta reservas en su capacidad de aportar al crecimiento económico del país. En este sentido estudios realizados por Martín (2014) brindan suficientes argumentos para afirmar que el sector turístico cubano presenta una situación de pérdida relativa de la competitividad en la región del Caribe. Para revertir esta situación es necesario eliminar, o al menos atenuar, el impacto negativo de las amenazas y las debilidades que inciden sobre su desempeño. Estos retos van desde la actual crisis económica mundial, hasta la concentración

\footnotetext{
* Universidad Central "Marta Abreu” de Las Villas, Santa Clara, Cuba; E-mail: dduffusm@uclv.edu.cu

**Universidad de Matanzas, Matanzas, Cuba; E-mail: roberto.pons@umcc.cu
} 
en el producto sol y playa, disminución del mercado europeo, oferta extrahotelera insuficiente, así como políticas de comercialización que han de perfeccionarse.

Referido a las políticas de comercialización, se considera que el turismo es un producto intangible que se comercializa a través de imágenes. En la literatura especializada se reconoce a la imagen de un hotel combinada con la imagen del destino y del país a que pertenece, como un inductor de compra determinante en el turismo (Beerli, Martin y Moreno, 2008; Perelló, 2014; Anholt, 2014). Por ello se espera que toda entidad perteneciente a este sector, mantenga una imagen positiva en el mercado. A pesar de contar con los medios para alcanzar la imagen deseada, en oportunidades no se logra este objetivo, lo que se debe fundamentalmente al manejo de esta variable desde una perspectiva teórica inconsistente.

Reflejo de esto, en la práctica empresarial la imagen, como regla, se trata como una variable exclusiva de la comunicación externa de los hoteles o cadena a que estos pertenecen. La misma se enfoca desde la proyección en los medios de comunicación, relegándose a un segundo plano la necesidad de conocer su configuración real en los clientes. En la mayoría de los casos no se toma en cuenta que la imagen tiene un sustrato objetivo que parte del conjunto de valores de uso que conforman el producto turístico y que se dan, en primera instancia, desde la producción. Lo que no excluye la influencia determinante del componente afectivo de la imagen en su configuración final.

En esta investigación se plantea que es necesario abordar la imagen desde la dirección estratégica, con el fin de proyectar estrategias coherentes para perfeccionar su proyección. Si se gestiona la imagen desde este punto de vista, se evitan las incongruencias entre la forma en que esta se comunica y la imagen que el visitante percibe durante su estancia en el hotel, lo que puede influir de manera negativa en su satisfacción. A esto se agrega que los procesos clave no siempre se planifican según las necesidades, deseos y expectativas de quienes los demandan; y como consecuencia se reducen las posibilidades de que sustenten una imagen positiva. Estos argumentos sintetizan la situación problemática de esta investigación, de donde se deriva como problema científico a resolver: ¿Cómo contribuir al logro de la imagen deseada en pequeños y medianos hoteles en Cuba a través de su evaluación en el proceso de dirección estratégica? Por tanto, el objetivo general de esta investigación radica en: Evaluar la imagen en el proceso de dirección estratégica en Cuba, mediante un procedimiento aplicado a pequeños y medianos hoteles en el sector turístico.

El presente estudio propone y valida un procedimiento para la evaluación de la imagen en el proceso de dirección estratégica, mostrando los resultados generales de su aplicación en 8 casos de estudios y los resultados detallados en uno de ellos: hotel Brisas Trinidad del Mar. Para ello se concibe la combinación de los métodos teóricos y empíricos. Del primer grupo se emplean el análisis-síntesis, inducción-deducción, abstracción-concreción, así como el histórico y lógico. Se consideran los métodos empíricos con un doble enfoque: cualitativo y cuantitativo en los cuales se emplean como técnicas: el análisis de contenido, observación directa, juicio de experto, dinámica de grupo, entrevista, encuesta, Matriz de análisis de rendimiento e importancia de los factores e identigrama. Para el análisis estadístico se emplean estadísticos de tendencia central, frecuencia, Kendall, Coeficiente de Correlación de Spearman, Coeficiente Tau-b de Kendall, Matriz de Correlación del Análisis Factorial, Exploración bivariante simultánea, Alpha de Cronbach, Índice de validez de contenido, Prueba de Mann Whitney y Kruskal Wallis. Para el procesamiento de la información se empleó el software estadístico SPSS vs- 21 y el software para la Evaluación de la Imagen de hoteles (SEIH vs-1), el cual se diseñó por la autora y un grupo de especialistas como soporte informatizado del procedimiento que se propone.

\section{Fundamentos teóricos de la investigación}

\subsection{La imagen de hoteles}

La evolución de la palabra imagen ha ido ampliando sus significaciones originarias, de tal modo que en la actualidad abarca un gran número de referentes; situación que tiene entre sus causas principales la polisemia del término, su ambigüedad semántica y una amplia vulgarización del mismo. Se consultaron definiciones relevantes, expresadas desde el año 1962 hasta 2015. Estos conceptos se concentran en cinco grupos, donde se distingue una corriente que conceptualiza la imagen como acontecimiento ficticio, otra referida a la imagen como una construcción de la empresa, otra como estereotipo, una cuarta referida a la imagen ícono, y un último grupo el cual alude a la imagen como un constructo mental (Boorstin, 1962; Echevarría, 2010; Eric y Mark, 2010; Arteaga, 2011; Manrrique y López, 2012; Ramírez et al., 2012). Los autores consideran este último enfoque el más acertado sobre la base de lo planteado en la teoría de la reflexión de los objetos por el pensamiento. 
La imagen del hotel implica la combinación del componente cognitivo y el componente afectivo, sobre la base objetiva de la realidad que se percibe; lo que puede involucrar la presencia de un componente único distintivo. Toda imagen deriva en determinada acción, por tanto se considera como otro componente de la misma: el conativo. Para conformar esta definición los autores tienen en cuenta los aportes realizados por: Pike y Ryan (2004), San Martín (2005), Costa (2008), Beerli et al. (2008), Capriotti (2013), Tanda (2011), Anholt (2014) en las cuales se consideran las diferencias del contexto histórico y el objeto de estudio a que las mismas se refieren.

Una reflexión acerca de la imagen que haga abstracción de todo elemento contingente, indica que esta es producto de factores subjetivos y objetivos. El proceso subjetivo es propio del sujeto en cuya psique se elabora la imagen, el cual responde a variables sociales, personales y psicológicas. Según investigaciones turísticas recientes acerca de las corrientes etnográficas y etnocéntricas en el estudio de la imagen, existe una tendencia universal a que las personas interpreten otras prácticas sociales desde la perspectiva de su país de origen (Perelló, 2014). Se considera que los factores objetivos que determinan la imagen de un hotel incluyen las estrategias de comunicación de la imagen concebidas en el país, destino y cadena corporativa a la que pertenezca el hotel, sus procesos clave, la identidad y comunicación de este tipo de establecimiento de alojamiento, donde intervienen también la calidad percibida y satisfacción del cliente sobre el servicio recibido. Los elementos antes mencionados abarcan el ámbito de la experiencia personal, las interrelaciones personales y las comunicaciones masivas.

Este modelo se diferencia de las tendencias que priman en la literatura especializada, las cuales identifican como elemento inductor principal en la formación de la imagen, a los procesos comunicacionales. En este modelo se pueden identificar los ámbitos descritos por Capriotti (2013) respecto a la formación de la imagen: las comunicaciones masivas, las interacciones personales recíprocas, y el ámbito de la experiencia personal en el hotel, donde lo objetivo es independiente del individuo en cuestión y actúa como inductor de todo proceso subjetivo (Duffus y Pons, 2014). Esta propuesta tiene como antecedentes las investigaciones de Sanz de la Tajada (1994); Muñiz (2009), Tanda (2011) y Capriotti (2013).

\subsection{La imagen en la dirección estratégica de hoteles}

En el turismo se identifica a la imagen como un activo intangible estratégico para la empresa hotelera y como un factor clave del éxito, ya que por medio de la imagen existe la entidad para los públicos objetivo y resulta un elemento determinante para su atracción hacia una decisión de compra positiva (Perelló, 2014). Para que el hotel logre ser percibido en sus públicos objetivo de la manera en que desea, se debe entender que la formación de determinada imagen es un proceso de interpretación acumulativa de información. Por consiguiente, no se actúa directamente sobre la imagen del hotel, sino sobre los factores que la determinan.

Los modelos de comunicación empresarial que predominan en la literatura científica y en la práctica profesional se caracterizan por dotar al emisor de un papel determinante en este proceso, donde el que posee la información es el elemento activo y con poder en la comunicación; mientras el receptor es completamente pasivo y manejable (Capriotti, 2013). En consecuencia, como generalidad, actualmente las estrategias que siguen los hoteles para el logro de la imagen deseada se limitan a la emisión de un mensaje que exprese cómo esperan que sus públicos objetivo perciban su realidad empresarial. La imagen se trata como una variable que el hotel puede manipular en su beneficio para atraer clientes. Esta posición se asume sobre la base de conceptualizaciones erróneas o limitadas de la imagen, donde se interpreta esta variable como ícono, acontecimiento ficticio o construcción de la empresa. Cuando se pretende proyectar una imagen sobre la base de mensajes que distorsionan la realidad del hotel, se corre el riesgo de afectar negativamente la satisfacción del cliente al experimentar, durante su estancia en el destino, una experiencia de engaño, ya que las expectativas creadas mediante el mensaje no pueden ser cumplimentadas con el servicio recibido. Las investigaciones actuales sobre la comunicación presionan a la modificación de esta concepción en el manejo de la imagen (Capriotti, 2013). El receptor ya no se enfoca como un elemento pasivo, sino que construye o reconstruye el mensaje enviado por el emisor a partir de su percepción de este respecto a su propia experiencia (Capriotti, 2013; Anholt, 2014). Aunque esta posición reconoce teóricamente la necesidad de que el mensaje sea diseñado y emitido en alineación con la realidad empresarial, no establece con claridad los nexos necesarios que se han de implementar entre la comunicación y otras funciones de la empresa para que esto suceda.

Se puede afirmar que el mensaje emitido es una condición necesaria para la conformación de la imagen deseada en los públicos objetivo, pero no suficiente. Es imprescindible que este mensaje sea reafirmado por la calidad con que se desarrollan los procesos clave, así como por la identidad transmitida 
por los empleados. Hasta el momento estas son áreas que se encuentran fragmentadas en el hotel. Sin embargo, la necesidad de lograr un impacto comunicativo fuerte en los turistas, sobre la base de una acción coordinada y coherente, hace indispensable que la comunicación de la organización se encuentre integrada al proceso de dirección estratégica que se lleve en el hotel (MINTUR, 2014). Para esto cada una de las diferentes partes objetivas que influyen en la conformación de la imagen y los elementos de la dirección estratégica que determinan la realidad del hotel, deben cumplir una función de apoyo y reafirmación de las demás, considerando la comunicación que se lleva a nivel de país, destino y cadena a que pertenezca la entidad.

\section{Metodología empleada}

Para suplir en la práctica empresarial este vacío teórico y metodológico respecto a la gestión de la imagen, los autores proponen un procedimiento desarrollado sobre la base de la experiencia de siete años de su aplicación en hoteles en Cuba. Esto ha permitido en distintas iteraciones corregir, adaptar y mejorar paulatinamente las características de los métodos de investigación empleados para satisfacer el objetivo de lograr la imagen deseada en hoteles (Duffus y Pons, 2014). En este sentido se introducen, en dicha propuesta metodológica los principales logros de 30 procedimientos concebidos para propósitos similares y se concibe en cada fase la eliminación de sus deficiencias. Las investigaciones más relevantes que sirven de referencia al procedimiento que se propone son: Chu y Choi (2000), Anholt (2003), Pike y Ryan (2004), Muñiz (2009), Tanda (2011), Martínez et al., (2012), Huawen (2015). El procedimiento propuesto consta de tres fases en las que se maneja información que se registra en 10 documentos que permiten su trazabilidad y uso eficiente.

\subsection{Fase I: Condiciones iniciales del hotel para la aplicación del procedimiento}

Esta fase tiene como objetivo determinar las características del hotel y su entorno, que se deberán tener en cuenta para la medición de su imagen. Para una mejor organización de esta fase la misma se divide en dos etapas. La primera etapa de esta fase ofrece la posibilidad de conocer el punto de partida para el tratamiento de este constructo, pues los atributos identificativos de la imagen del país, destino y la cadena a la que pertenece este tipo de establecimiento de alojamiento, influirán en la percepción general de la misma. Además, la etapa satisface la necesidad de tipificar el hotel para escoger los atributos correctos con el fin de medir su imagen, a lo que se agrega determinar sus posibilidades económicas y financieras para enfrentar futuros cambios necesarios en sus estrategias. La segunda etapa tiene como objetivo determinar las características y limitaciones del proceso de dirección estratégica que se lleva a cabo en el hotel para introducir en el mismo la evaluación de la imagen en función de lograr su estado deseado. Durante la Fase I se propone emplear como técnicas para obtener y procesar la información, el análisis de documentos, el Muestreo Aleatorio Simple, la estadística descriptiva y el análisis vertical de razones.

\subsection{Fase II: Evaluación de la imagen del hotel}

La Fase II persigue obtener como resultado la medición de la imagen del hotel de forma válida y fiable considerando la evaluación de los factores que influyen sobre el logro de su estado deseado. Para ello se parte de la caracterización de los atributos bases de la promoción del hotel. Además se considera la propuesta de dimensiones y atributos para la descripción de la imagen. Estas se determinan según las dimensiones genéricas para la tipología a que corresponde el hotel según categoría y localización. Estas dimensiones se reflejan en una encuesta que constituye uno de los instrumentos para medir este constructo, las que se adaptan a las características particulares del establecimiento objeto de estudio donde se valora su validez y fiabilidad. Concluida esta fase se ofrece un perfil de la imagen primaria ${ }^{1}$ del hotel sobre la base de la valoración de la realidad de este por sus clientes, donde se identifican los elementos de identidad y de la comunicación externa influyentes.

Para una mejor organización de esta fase, la misma se ha dividido en 7 etapas. Estas etapas comprenden: la identificación de los atributos de comunicación externa, el desarrollo y validación de dimensiones y atributos genéricos; el ajuste de los instrumentos propuestos a las condiciones de cada hotel; la medición de la identidad e imagen; el análisis de fiabilidad de los instrumentos aplicados, así como el procesamiento y análisis de los datos resultantes de la medición. Todos los hoteles donde se aplica este procedimiento no poseen las mismas condiciones. De ahí que estas etapas sean aplicadas según las características que presenta el objeto de análisis. 
Durante esta Fase II se emplean como técnicas para obtener y procesar la información el análisis de contenido, el muestreo por cuotas, el muestreo Aleatorio Estratificado de tipo proporcional, el análisis de documentos, el juicio de expertos, la observación directa, la dinámica de grupo, la encuesta, la entrevista, estadística descriptiva e inferencial y el Sistema de Evaluación de la Imagen del Hotel (SEIH vs.1), software creado por la autora en conjunto con un equipo multidisciplinario de profesionales de Universidad Central "Marta Abreu" de Las Villas.

\subsection{Fase III: Incorporación de los resultados de la medición de la imagen al proceso de dirección estratégica en el hotel}

Esta fase se caracteriza por poseer 4 etapas, en las que se establecen comparaciones para determinar diferencias entre elementos que deberían funcionar armónicamente. Tal es el caso de la imagen secundaria ${ }^{2}$ con la imagen primaria; la imagen con la identidad, así como el perfil estratégico del hotel y el perfil que incluye las oportunidades, amenazas, fortalezas y debilidades, detectadas por empleados y clientes. En la Fase III se propone formular los cambios pertinentes en el análisis interno y externo, para incluir las perspectivas de los empleados y clientes en la determinación del rumbo estratégico del hotel enfocado a sustentar la imagen deseada. Para obtener y procesar la información en esta fase se emplea el ideograma, el identigrama, el imagograma y la matriz de rendimiento-importancia del factor.

Aunque la dirección estratégica es un proceso que abarca el largo plazo y además la imagen cambia lentamente; es necesario controlar el cambio sistemáticamente. En consecuencia, se propone aplicar este procedimiento anualmente. $\mathrm{Al}$ considerarse el tablero de mando dentro de este proceso, se concibe la imagen como indicador de cliente; donde su medición sistemática permite detectar avances o retrocesos en el trayecto para lograr la imagen deseada, así como en el logro del enfoque integrado de orientación al cliente.

\section{Resultados y Discusión}

\subsection{Validación del procedimiento propuesto}

Para comprobar que los métodos propuestos para lograr la imagen deseada en hoteles en Cuba a través de su evaluación en la dirección estratégica son válidos y fiables se sometió el procedimiento al criterio de expertos y se aplicó en 7 hoteles de Cuba en 8 ocasiones (Ver Tabla 1).

El estudio de la respuesta de los expertos (Tabla 2) permite afirmar según el grado de significación que muestra el coeficiente de $\mathrm{W}$ de Kendall, que existe concordancia entre los criterios emitidos por los expertos y esta es alta. Al corroborar estos resultados, según la significación de la prueba Kruskal Wallis se comprueba que no hay contradicciones entre los argumentos dados por los expertos respecto a la presencia de ninguna de las características evaluadas para el procedimiento. Se considera el criterio que más predomina entre estos, expresado por la moda; así se puede afirmar que el procedimiento implementa cabalmente el modelo teórico que lo sustenta y al mismo tiempo es capaz de cumplir con los objetivos que se propone. Los resultados obtenidos mediante la estadística descriptiva, se comprueban con el cálculo del Índice de Validez de Contenido (IVC) el cual fue adaptado de la propuesta realizada por (Lawshe, 1975) donde la adecuación de todas las características descritas al procedimiento propuesto son evaluadas de positivas. Aunque el procedimiento obtuvo buena evaluación de los jueces en sentido general, este debe estar en continua revisión; ya que aspectos como la estructuración y el carácter participativo deben mejorarse, según lo que expresa la media, la frecuencia relativa y el IVC. Se puede afirmar que la encuesta utilizada por los expertos para validar el procedimiento posee consistencia interna, por lo que los resultados que se derivan de ella son fiables. 
Tabla 1: Ficha técnica de la investigación

\begin{tabular}{|c|c|c|c|}
\hline Universo & Empleados & Turistas & Expertos \\
\hline Unidad muestral & 529 & 866 & 15 \\
\hline $\begin{array}{c}\text { Procedimiento de } \\
\text { muestreo }\end{array}$ & $\begin{array}{c}\text { Aleatorio estratificado de tipo } \\
\text { proporcional: según ocupación. }\end{array}$ & $\begin{array}{c}\text { Aleatorio } \\
\text { estratificado de } \\
\text { tipo proporcional: } \\
\text { según } \\
\text { nacionalidad. }\end{array}$ & $\begin{array}{c}\text { Método propuesto } \\
\text { por Cyret y March } \\
(1965)\end{array}$ \\
\hline Error muestral & $9 \%$ & $9 \%$ & $9 \%$ \\
\hline Nivel de confianza & $95 \%$ & $95 \%$ & $95 \%$ \\
\hline $\begin{array}{c}\text { Fecha de trabajo } \\
\text { de campo }\end{array}$ & enero de 2009- diciembre de 2014. & \\
\hline
\end{tabular}

Fuente: Elaboración propia

Tabla 2: Características del juicio de expertos respecto al procedimiento propuesto

\begin{tabular}{|c|c|c|c|c|c|c|c|}
\hline \multirow[b]{2}{*}{$\begin{array}{l}\text { Características del } \\
\text { procedimiento }\end{array}$} & \multirow[b]{2}{*}{ Media } & \multirow[b]{2}{*}{ Moda } & \multicolumn{5}{|c|}{ Frecuencia Relativa (\%) } \\
\hline & & & $\begin{array}{l}\text { Nivel medio } \\
\text { de adecuación }\end{array}$ & Adecuado & $\begin{array}{c}\text { Muy } \\
\text { adecuado }\end{array}$ & IVC & $\begin{array}{c}\text { Kruskal } \\
\text { Wallis }\end{array}$ \\
\hline Adecuación al modelo & 4.80 & 5 & 0 & 20.0 & 80.0 & 1 & 0.459 \\
\hline Carácter sistémico & 4.73 & 5 & 0 & 26.7 & 73.3 & 1 & 0.196 \\
\hline Consistencia lógica & 4.33 & 4 & 0 & 66.7 & 33.3 & 1 & 0.186 \\
\hline Participativo & 4.20 & $4^{3}$ & 20.0 & 40.0 & 40.0 & 0.6 & 0.312 \\
\hline Flexible & 4.40 & 4 & 0 & 60.0 & 40.0 & 1 & 0.540 \\
\hline $\begin{array}{l}\text { Mejoramiento } \\
\text { continuo }\end{array}$ & 4.67 & 5 & 0 & 33.3 & 66.7 & 1 & 0.079 \\
\hline Valor metodológico & 4.53 & 5 & 0 & 46.7 & 53.3 & 1 & 0.939 \\
\hline Viabilidad & 4.27 & 4 & 0 & 73.3 & 26.7 & 1 & 0.108 \\
\hline Estructuración & 3.87 & 4 & 13.3 & 86.7 & 0 & 0.73 & 0.546 \\
\hline Racionalidad & 4.53 & 5 & 0 & 46.7 & 53.3 & 1 & 0.126 \\
\hline $\begin{array}{l}\text { Cumplimiento de } \\
\text { objetivos }\end{array}$ & 4.73 & 5 & 0 & 26.6 & 73.4 & 1 & 0.385 \\
\hline $\begin{array}{l}\text { significación W de } \\
\text { Kendall: } 0.000 \text { Alpha } \\
\text { de Cronbach }=0.726\end{array}$ & & & & & & & \\
\hline
\end{tabular}

Fuente: Elaboración propia

Resultado de la aplicación del procedimiento en ocho ocasiones en siete hoteles de Cuba se pudo comprobar el impacto de este sobre el índice de satisfacción. Se selecciona el índice de satisfacción para medir el impacto del procedimiento debido a que este es un indicador externo a la propuesta metodológica que se realiza y está directamente relacionado con la variable principal en la misma (Chu y Choi, 2000; Beerli et al., 2008).

La satisfacción tiene una relación directa y fuerte con la congruencia entre imagen secundaria e imagen primaria del hotel (Chu y Choi, 2000; Beerli et al., 2008 ). De allí que luego de aplicarse las acciones que se proponen en el procedimiento para estos hoteles, un indicador que muestra el impacto de este en el logro de la imagen deseada es el índice de satisfacción. Los investigadores reconocen que 
existen elementos psicosociales no controlables que pueden influir en los resultados que se muestran, pero consideran que no demeritan la calidad de la demostración. El año antes de la aplicación del procedimiento, la dirección de los hoteles parte de la muestra no implementó otras estrategias diferentes de las que se proponen para sustentar una imagen positiva.

En el 100\% de los casos en que se aplica el procedimiento para la evaluación de la imagen en la dirección estratégica de hoteles en Cuba, se logra un aumento en el índice de satisfacción; por consiguiente es posible afirmar que el procedimiento mejora la percepción de la imagen en los clientes, considerando la relación directa y fuerte entre la imagen y la satisfacción.

\subsection{Aplicación integral del procedimiento al caso de estudio: hotel Brisas Trinidad del Mar}

Mediante el análisis detallado del caso de estudio hotel Brisas Trinidad del Mar, se demuestra que el procedimiento para la evaluación de la imagen en la dirección estratégica de hoteles en Cuba que se propone, es capaz de ofrecer los resultados que declara.

\subsubsection{Fase I: Condiciones iniciales del hotel para la aplicación del procedimiento}

El Country Brand Index ubica a Cuba en el número 9 en el ranking de América Latina y a nivel mundial en el puesto 56, donde ha retrocedido en 6 posiciones. El hotel Brisas Trinidad del Mar está enclavado en el destino Trinidad, el cual tiene una imagen afianzada a nivel internacional como Ciudad Museo del Mar Caribe. Cuenta con 25 atractivos inventariados entre los sitios web: Infotur, La turística, Tripadvisor y Hicuba. De estos atractivos el $76 \%$ son socioculturales y el $24 \%$ naturales. El entorno competitivo se caracteriza ser el 90\% del hospedaje registrado de tipo hostal. En el sitio web Tripadvisor se registra una serie de opiniones donde el $90 \%$ son favorables al destino.

Este establecimiento de alojamiento pertenece a la Cadena Cubanacán, grupo líder en la actividad turística cubana y recibe cerca del $40 \%$ del turismo internacional que visita a Cuba. Al considerar la información que se proyecta en el sitio web oficial de Cubanacán S.A., esta promueve su imagen secundaria en base a: experiencia de sus profesionales, acertada calidad de los servicios, variedad de la oferta y variedad de productos.

La imagen percibida por los clientes luego de su visita a las instalaciones de Cubanacán se determinó a través del sitio web Tripadvisor. Mediante un muestreo aleatorio simple considerando la población los 76 hoteles de esta cadena, con un error del $15 \%$ se evalúa la imagen primaria de la cadena a que pertenece el hotel, la cual se caracteriza por ser heterogénea, e incluir desde hoteles calificados como malos (11\%), hasta excelentes (14\%), la evaluación que más se repite es de muy bueno (55\%), por lo que se considera que la imagen general de esta cadena es positiva. No obstante, existen dificultades que inciden negativamente en su imagen, las que se relacionan con las habitaciones, el servicio y la relación calidad-precio.

El hotel Brisas Trinidad del Mar es un hotel de playa con categoría de 4 estrellas. Posee un total de 187 empleados y 241 habitaciones. Teniendo en cuenta el acceso a la información y la pertinencia de la misma para la investigación; se consideró suficiente el cálculo de la solvencia, y rentabilidad general del hotel para conocer su situación económica-financiera de manera general, la cual es favorable.Dentro de la valoración del proceso de dirección estratégica en el hotel resaltan como aspectos negativos que en el proceso de diseño estratégico, la empresa parte de estrategias corporativas sin antes definir con claridad los resultados que estas persiguen.

\subsubsection{Fase II: Evaluación de la imagen del hotel}

Para caracterizar la imagen secundaria del hotel Brisas Trinidad del Mar se utiliza como método el análisis de contenido en medios de promoción a través de Internet. Según esto, se puede caracterizar el corpus comunicativo que sustenta una parte importante de la imagen secundaria de este hotel, como se muestra en la tabla 3. Prima el estilo de redacción informativo sobre la explotación del vocabulario promocional, esto incide en que no existan las suficientes alusiones al componente afectivo de la imagen. En ninguno de los medios se resaltan elementos distintivos. El 90\% de lo que se transmite en el corpus de comunicación analizado, se basa en atributos del componente cognitivo. Dentro de este, el $24 \%$ de lo que se dice se refiere a una descripción de las facilidades dentro de las habitaciones y el $44 \%$ se enfoca a describir en detalles todos los servicios y facilidades complementarios que soportan la categoría del hotel. Los elementos visuales de la imagen, los íconos identificativos y las fotografías podrían emplearse en mayor medida. 


\section{Tabla 3: Imagen secundaria del hotel Brisas Trinidad del Mar}

\begin{tabular}{|l|c|}
\hline Componentes de la imagen & Presencia en el corpus comunicativo \\
\hline 1. Componente único distintivo & $0 \%$ \\
\hline 2. Componente afectivo & $2 \%$ \\
\hline 3. Componente cognitivo: & $90 \%$ \\
\hline 3.a. Dimensión Servicios generales & $3 \%$ \\
\hline 3.b. Dimensión Habitaciones & $24 \%$ \\
\hline 3.c. Dimensión Gastronomía, bebidas y recreación & $22 \%$ \\
\hline 3.d. Dimensión Servicios e instalaciones complementarias & $44 \%$ \\
\hline 3.e. Dimensión Condiciones del Destino & $7 \%$ \\
\hline 4. Aparición de íconos distintivos & $1 \%$ \\
\hline 5. Fotos del hotel & $8 \%$ \\
\hline
\end{tabular}

Fuente: Elaboración propia

Los instrumentos para medir la identidad e imagen del hotel Brisas Trinidad del Mar fueron aprobados para su aplicación por el Consejo de dirección ampliado del Complejo Hotelero. De acuerdo con la técnica de trabajo en grupo a través de la dinámica de grupo nominal se logró un consenso entre los implicados. Este paso tiene como objetivo ajustar los componentes y atributos de la imagen a las características específicas del hotel objeto de estudio. Dentro de los elementos sujetos a cambios están la inclusión de posibles respuestas para identificar el componente único distintivo del hotel. Cada uno de estos elementos permite hacer la pregunta 1 del cuestionario semiabierta, como sugieren los expertos. En el componente cognitivo se incorporan como atributos la valoración del servicio de spa, como un interés particular de la dirección del complejo.

A los cuestionarios con los atributos genéricos se les realizan las adecuaciones y sobre esta base se perfilan las preguntas de cada cuestionario y se someten a un respondent debriefing, forma de pretest donde se escogen a 16 empleados y 20 turistas.

Teniendo cuestionarios donde se ha demostrado su validez, adecuación a las características del hotel y posibilidad de ser comprendidos por la población objeto de estudio, se pasa entonces a su aplicación. Las características del muestreo y la recogida de datos se resumen en la ficha técnica de la investigación (Tabla 4).

\section{Tabla 4: Ficha técnica de la investigación en el hotel Brisas Trinidad del Mar}

\begin{tabular}{|l|c|c|}
\hline Universo & Empleados & Turistas \\
\hline Población & 176 empleados & 452 plazas \\
\hline Unidad muestral & $\mathrm{n}=90$ & $\mathrm{n}=106$ \\
\hline $\begin{array}{l}\text { Métodos de obtención de } \\
\text { información }\end{array}$ & Entrevista estandarizada cerrada & $\begin{array}{c}\text { Encuesta personal de } \\
\text { autoaplicación }\end{array}$ \\
\hline Procedimiento de muestreo & $\begin{array}{c}\text { Aleatorio estratificado de tipo } \\
\text { proporcional: según ocupación. }\end{array}$ & $\begin{array}{c}\text { Aleatorio estratificado de tipo } \\
\text { proporcional: según nacionalidad. }\end{array}$ \\
\hline Error muestral & $9 \%$ & $9 \%$ \\
\hline Nivel de confianza & $95 \%$ & $95 \%$ \\
\hline
\end{tabular}

Fuente: Elaboración propia

Se introducen los datos resultantes de las encuestas a empleados y clientes en el software SEIH vs $1^{4}$. $\mathrm{Al}$ calcularse el estadígrafo Alpha de Cronbach a los cuestionarios a empleados y clientes se demostró que estos tienen una consistencia interna de 0.894 y 0.953 respectivamente, por lo que los resultados que se obtienen de su aplicación son fiables. 
Los datos resultantes de la medición de la identidad e imagen se resumen a continuación en las tablas de la 5 a la 9. Considerando el criterio de empleados y clientes, se puede afirmar que el hotel tiene un componente que lo distingue del resto de sus similares: la arquitectura del hotel, la que simula a la ciudad de Trinidad (Tabla 5).

Tabla 5: Características del componente único-distintivo del hotel brisas trinidad del mar

\begin{tabular}{|l|c|c|}
\hline Componente único-distintivo & Imagen (\%) & Identidad (\%) \\
\hline Arquitectura del hotel & 48.1 & 46.7 \\
\hline Arquitectura del hotel y servicio prestado & 17 & 14.4 \\
\hline Arquitectura del hotel servicio prestado y ubicación & 9.4 & 6.7 \\
\hline Arquitectura del hotel ubicación geográfica & 25.5 & 32.2 \\
\hline
\end{tabular}

Fuente: Elaboración propia a partir del software SEIH vs1.

El ambiente que suscita el hotel Brisas Trinidad del Mar es muy agradable, muy tranquilo, muy relajado, muy seguro y muy atrayente, según los clientes y empleados. Ambas poblaciones encuestadas coinciden en que otro adjetivo que describe el componente afectivo de este hotel es excitante Tabla 6.

Tabla 6: Características del componente afectivo del hotel Brisas Trinidad del Mar

\begin{tabular}{|l|c|c|c|c|}
\hline \multirow{2}{*}{ Componente Afectivo } & \multicolumn{2}{|c|}{ Imagen } & \multicolumn{2}{c|}{ Identidad } \\
\cline { 2 - 5 } & Media & Moda & Media & Moda \\
\hline ambiente desagradable-agradable & 4.60 & 5 & 4.56 & 5 \\
\hline ambiente aburrido-excitante & 3.86 & 4 & 3.76 & 4 \\
\hline ambiente ruidoso-tranquilo & 4.29 & 5 & 4.23 & 5 \\
\hline ambiente tenso-relajado & 4.44 & 5 & 4.48 & 5 \\
\hline ambiente inseguro-seguro & 4.76 & 5 & 4.72 & 5 \\
\hline ambiente Indiferente-atrayente & 4.60 & 5 & 4.56 & 5 \\
\hline
\end{tabular}

Fuente: Elaboración propia a partir del software SEIH vs1.

El componente cognitivo se describe de manera resumida según las dimensiones que lo conforman en la tabla 7. Es significativo destacar que todos los atributos fueron evaluados de muy importantes por empleados y clientes. Los dos grupos encuestados consideran que todas las dimensiones están evaluadas de manera positiva, excepto en el caso de los clientes que encuentran una serie de deficiencias relacionadas con la dimensión Habitaciones. La calificación anterior no excluye que ambas poblaciones indiquen otros atributos en diversas dimensiones con evaluaciones desfavorables. 

Tabla 7: Características del componente cognitivo por
dimensiones del hotel Brisas Trinidad del Mar

\begin{tabular}{|l|c|c|c|c|}
\hline \multirow{2}{*}{ Componente Cognitivo } & \multicolumn{2}{|c|}{ Imagen } & \multicolumn{2}{c|}{ Identidad } \\
\cline { 2 - 5 } & Media & Moda & Media & Moda \\
\hline Servicios generales & 3.92 & 4 & 3.70 & 4 \\
\hline Habitaciones & 3.22 & 3 & 3.65 & 4 \\
\hline Gastronomía, Bebidas y Recreación & 3.92 & 4 & 3.79 & 4 \\
\hline Servicios e instalaciones complementarias & 3.92 & 4 & 3.79 & 4 \\
\hline Condiciones del destino & 3.57 & 4 & 3.90 & 4 \\
\hline
\end{tabular}

Fuente: Elaboración propia a partir del software SEIH vs1.

En la encuesta a los empleados, estos unánimemente opinan que con toda certeza sus clientes regresarían al hotel ya que se cumplieron totalmente sus expectativas, último aspecto con el que no todos coinciden. Por otra parte, la imagen del hotel se considera como positiva por la mayoría de los empleados, que de igual forma valoran su contribución al logro de la misión y visión del hotel Brisas Trinidad del Mar (Tabla 8).

Tabla 8: Autoevaluación de los empleados desde un enfoque de identidad

\begin{tabular}{|c|c|c|c|c|}
\hline Empleados & Media & Moda & Mínimo & Máximo \\
\hline Repetirían sus clientes la visita & 5 & 5 & 5 & 5 \\
\hline Percepción de la imagen general por los empleados & 3.97 & 4 & 2 & 5 \\
\hline Cree que sus clientes cumplen sus expectativas & 4.24 & 5 & 2 & 5 \\
\hline Contribución a la misión y la visión & 3.84 & 4 & 2 & 5 \\
\hline
\end{tabular}

Fuente: Elaboración propia a partir del software SEIH vs1.

La mayoría de los clientes opinan que con toda certeza regresarían al hotel teniendo estos la máxima apreciación de la imagen general de este y un nivel positivo respecto al cumplimiento de sus expectativas (Tabla 9).

Tabla 9: Satisfacción, componente conativo e imagen en general del cliente

\begin{tabular}{|l|c|c|c|c|}
\hline Clientes & Media & Moda & Mínimo & Máximo \\
\hline Intención de repetir la visita & 4.78 & 5 & 3 & 5 \\
\hline Percepción de la imagen general & 3.93 & 5 & 1 & 5 \\
\hline Cumplimiento de las expectativas & 3.98 & 4 & 2 & 5 \\
\hline
\end{tabular}

Fuente: Elaboración propia a partir del software SEIH vs1.

No existen diferencias significativas en cuanto a la percepción de la imagen general que poseen los empleados según las variables de segmentación empleadas. En el caso de los clientes existen diferencias significativas entre los criterios que se emiten por los clientes respecto a la imagen general del hotel. Estas discrepancias se encuentran entre los rangos de edades así como entre los clientes con distinta frecuencia de visita al hotel. Se ha de observar que el grupo de personas entre 55 y 64 años de edad son los que tienen mayor cantidad de criterios desfavorables con respecto a la imagen del hotel. Las mejores opiniones se encuentran en los clientes entre 25 y 34 años. Respecto a la frecuencia con que han visitado el hotel, los que lo hacen entre dos y tres veces tienen mayor cantidad de opiniones en 
detrimento de la imagen general del hotel con respecto a los que visitan la instalación por primera vez o en cuatro o más ocasiones. Para conocer cómo valoran los componentes de la imagen estos grupos se realiza una exploración bivariante simultánea (Tablas 10 y 11).

Tabla 10: Diferencias en la percepción de la imagen general según rangos de edades

\begin{tabular}{|l|c|c|c|c|c|c|}
\hline Criterios respecto a la imagen & $\begin{array}{c}\text { menos } \\
\mathbf{d e ~ 2 5}\end{array}$ & $\mathbf{2 5 - 3 4}$ & $\mathbf{3 5 - 4 4}$ & $\mathbf{4 5 - 5 4}$ & $\mathbf{5 5 - 6 4}$ & $\begin{array}{c}\text { más de } \\
\mathbf{6 4}\end{array}$ \\
\hline Opiniones negativas & $40 \%$ & $0 \%$ & $31 \%$ & $47 \%$ & $55 \%$ & $37 \%$ \\
\hline Opiniones positivas & $60 \%$ & $100 \%$ & $69 \%$ & $53 \%$ & $45 \%$ & $63 \%$ \\
\hline Prueba Kruskal Wallis & $\begin{array}{c}\text { Sig. } \\
0,018\end{array}$ & & & & & \\
\hline
\end{tabular}

Fuente: Elaboración propia a partir del software SEIH vs1.

Tabla 11: Diferencias en la percepción de la imagen general según frecuencia de visita

\begin{tabular}{|l|c|c|c|}
\hline Criterios respecto a la imagen & primera vez & 2-3 veces & 4 o más veces \\
\hline Opiniones negativas & $26 \%$ & $36 \%$ & $26 \%$ \\
\hline Opiniones positivas & $74 \%$ & $64 \%$ & $74 \%$ \\
\hline Prueba Kruskal Wallis & Sig. 0,001 & & \\
\hline
\end{tabular}

Fuente: Elaboración propia a partir del software SEIH vs1.

\subsubsection{Fase III. Incorporación de la medición de la imagen al proceso de dirección estratégica en el hotel}

Según la ficha de contenido que describe los atributos de promoción del hotel en Internet y considerando los resultados de la medición de las percepciones de los clientes respecto a los procesos clave del hotel, se detectan incongruencias entre la imagen secundaria y la imagen primaria del hotel Brisas Trinidad del Mar.

No existen diferencias entre lo que opina la mayoría de los clientes y empleados respecto al componente único distintivo de la imagen, al componente afectivo, al componente conativo y el grado de cumplimiento de las expectativas de los clientes. Por otra parte, respecto al componente cognitivo existen coincidencias entre la evaluación otorgada por la mayoría de ambos grupos encuestados. Excepto en el caso de las Habitaciones, que mientras los clientes las evalúan desfavorablemente, los empleados entienden positivo el aporte de este atributo a la imagen general.

Al considerar el imagograma e ideograma del hotel Brisas Trinidad del Mar, así como sus condiciones iniciales, se propone el identigrama que se muestra. El mismo se proyecta teniendo en cuenta que no se deben hacer, por el momento, cambios en la proyección de la identidad respecto al componente afectivo. Sí se considera que el componente cognitivo, la cultura organizacional, y la filosofía de dirección deben girar en torno al logro de la excelencia en las dimensiones: gastronomía, bebidas y recreación, así como en los servicios e instalaciones complementarias. Para ello se cuenta con condiciones financieras y una percepción favorable al cambio. De igual forma, es imprescindible proyectar cambios respecto a las condiciones de las habitaciones.

Cuando se comparó el perfil estratégico elaborado por el hotel con el perfil estratégico ampliado sobre la base de la identidad e imagen se pudo detectar que todas las fortalezas identificadas por el hotel se corresponden con fortalezas identificadas por los empleados y clientes. No obstante, es posible incorporar al perfil estratégico del hotel, 3 fortalezas que según los clientes no han sido consideradas en el diagnóstico estratégico. Según los clientes encuestados, existen 16 aspectos de los procesos clave del hotel, que aunque tienen un desempeño positivo se pueden mejorar. Los directivos del hotel identifican como debilidad el estado técnico de las habitaciones, aspecto que corroboran clientes y empleados. Se describe como otra debilidad en el análisis interno del hotel la calidad del servicio, pero no se identifican 
los aspectos que más influyen en esta. Cuando se analizó la imagen percibida se pudo constatar que sobre este aspecto tributan de forma negativa, al menos, la falta de atención personalizada, estado técnico de las habitaciones y las insuficientes ofertas del minibar en las habitaciones. Esto no excluye la influencia negativa que pueden tener los elementos citados como atributos susceptibles de mejora. Aunque el hotel identifica como una debilidad las ofertas gastronómicas, los clientes y empleados reconocen que son aspectos a mejorar pero que no constituyen un determinante para la valoración negativa de la imagen. En el análisis de las oportunidades detectadas se deben incluir otras cinco que identifican clientes y empleados; también se debe tener en cuenta que la oferta extrahotelera constituye una amenaza para la percepción de la imagen en los clientes de Brisas Trinidad del Mar. Las incongruencias descritas entre el análisis DAFO del hotel y la percepción de la identidad e imagen de empleados y clientes respectivamente, pueden ser el origen de una toma de decisiones ineficaces, ya que estas no responden totalmente a elementos soportes de la estrategia empresarial como son los clientes, valores compartidos y cultura organizacional; en consecuencia se torna difícil que las acciones cotidianas contribuyan al logro de la imagen deseada.

Para la elaboración de objetivos y estrategias en el hotel Brisas Trinidad del Mar se deben considerar los aspectos que se enumeran a continuación4. Los mismos implican decisiones que han de ser gestionadas de manera oportuna y consecuente a nivel del hotel, complejo, cadena o destino. A continuación se muestran las directrices estratégicas a implementar en el hotel como resultado de la aplicación de este procedimiento.

Directriz estratégica \# 1: Reducción de las incongruencias entre la imagen secundaria del hotel y la imagen primaria. Línea de acción 1.1: Incluir como elemento único distintivo una descripción de la arquitectura peculiar del hotel, en la imagen secundaria que se promociona de Brisas Trinidad del Mar en el corpus comunicativo en internet, así como aludir al ambiente que se puede experimentar en el mismo según los resultados del componente afectivo de la imagen. Línea de acción 1.2: Se deben eliminar las alusiones positivas sobre las habitaciones, limitándose a su descripción. La promoción debe hacer énfasis en aspectos relacionados con la gastronomía, bebidas y recreación, así como a los servicios y facilidades adicionales del hotel. Línea de acción 1.3: Incorporar en los medios de comunicación como forma de promoción la opción de visualizar las opiniones de los clientes respecto al hotel.

Directriz estratégica \# 2: Reducción de las incongruencias entre la identidad y la imagen primaria. Línea de acción 2.1: Se debe trabajar para que los empleados incorporen a sus valores compartidos la importancia de una atención personalizada, un trato amable a los clientes, una higiene óptima, así como una actitud colaborativa respecto a la recreación. Estos aspectos se deben concebir desde la filosofía empresarial hasta la cultura organizacional que se propicia de manera intencionada por los directivos. Línea de acción 2.2: La imagen del hotel es mejor valorada por los clientes que por los empleados, estos últimos consideran que el servicio del hotel no se corresponde con la categoría. En este sentido es importante incluir este aspecto como una debilidad en el análisis DAFO e implementar, con la participación de los empleados, las medidas para revertir esta situación.

Directriz estratégica \# 3: Reducción de las incongruencias entre la percepción de la realidad relevante a la imagen por clientes versus percepción de los directivos. Línea de acción 3.1: Mantener el trabajo que se realiza respecto a la capacitación de los empleados, el servicio rápido, servicio de registro y salida, servicio de información turística, servicios especializados para niños, el surtido de las tiendas, servicios de caja fuerte, así como los servicios de peluquería, barbería y spa. Línea de acción 3.2: Concentrar las acciones en la mejora de: higiene, climatización, decoración de las habitaciones, así como su capacidad para que estas sean confortables y acogedoras, debido a que esta dimensión es la peor evaluada. Aunque, según los clientes, existen 17 aspectos que se deben perfeccionar. Línea de acción 3.3: Tratar, en el diagnóstico estratégico del hotel, los aspectos referidos a la gastronomía como elementos a mejorar y no como debilidades. Línea de acción 3.4: La debilidad prioritaria a solucionar es el estado técnico de las habitaciones. Aunque se deben eliminar, también, los aspectos negativos identificados con respecto a la insuficiente oferta en el minibar, así como las deficiencias en la atención personalizada. Línea de acción 3.5: Considerar acciones que atenúen el impacto de la insuficiente oferta extrahotelera. Línea de acción 3.6: Se debe trabajar en solucionar aspectos que identifican como negativos los clientes; aunque la imagen primaria del hotel es muy positiva, existen criterios, no predominantes, totalmente negativos respecto a esta variable. Línea de acción 3.7: Al realizar una exploración bivariante simultánea, para las personas entre 55 y 64 años se detectó que uno de los elementos que influye de manera negativa en su percepción general de la imagen es la recreación nocturna, por lo que se deben concebir opciones que incluyan los gustos de este segmento de clientes. 


\subsubsection{Control de la imagen deseada en el hotel Brisas Trinidad del Mar desde la Fase III del procedimiento}

Los autores entienden que la imagen depende al menos de elementos de índole subjetivo propios del contexto psicosocial del individuo, así como de la realidad que este percibe en el hotel a través de la evaluación de los procesos clave, la identidad y comunicación. En la Fase III del procedimiento se determinan las incongruencias entre la imagen secundaria y la imagen primaria; entre la identidad y la imagen primaria, así como entre la percepción de la realidad del hotel que influye en la imagen que poseen los clientes y las perspectivas de valoración de los directivos respecto a estos elementos. En consecuencia, a medida que estas incongruencias se reducen existen mayores posibilidades de que el hotel se acerque a la imagen deseada, la cual es un proceso iterativo en el tiempo, consecuencia de acciones sistemáticas de influencia sobre los factores que la determinan. En la Tabla 12 se muestran datos referidos a la aplicación en el hotel Brisas Trinidad del Mar del procedimiento para la evaluación de la imagen en la dirección estratégica de hoteles en Cuba en el año 2009, 2016. En este período de tiempo se observan mejorías en la percepción de la imagen general del hotel por parte de los públicos externos, además se reducen las incongruencias existentes entre los elementos que determinan la formación de la imagen deseada; por lo que se puede afirmar que la aplicación del procedimiento permite lograr su propósito.

\section{Tabla 12: Comportamiento en el tiempo de la imagen del hotel Brisas Trinidad del Mar y de los factores que influyen en esta.}

\begin{tabular}{|c|c|c|}
\hline Indicadores & Año 2009 & Año 2016 \\
\hline Valoración de la imagen general (valor medio). & 3.80 & 4.2 \\
\hline $\begin{array}{l}\text { Incongruencias: imagen secundaria versus imagen primaria (ámbito } \\
\text { de las comunicaciones masivas). }\end{array}$ & $60 \%$ & $38 \%$ \\
\hline $\begin{array}{l}\text { Incongruencias: imagen primaria versus identidad } \\
\text { (ámbito de las interacciones personales recíprocas). }\end{array}$ & $85 \%$ & $35 \%$ \\
\hline $\begin{array}{l}\text { Incongruencias: percepción de la realidad relevante a la imagen por } \\
\text { clientes versus directivos (ámbito de la experiencia personal en el } \\
\text { hotel). }\end{array}$ & $54 \%$ & $10 \%$ \\
\hline
\end{tabular}

Fuente: Elaboración propia.

\section{Conclusiones}

$\mathrm{Al}$ analizar críticamente las concepciones teóricas y metodológicas expuestas en la ciencia hasta el momento acerca del tratamiento de la imagen de empresas, en esta investigación se establece que los factores objetivos determinan el logro de la imagen deseada en un hotel. Estos se relacionan con diversas funciones de la empresa, por lo que se entiende necesario abordar esta variable desde la dirección estratégica para buscar una complementariedad entre las estrategias de comunicación externa y las estrategias relacionadas con la consolidación de la identidad del hotel y el logro de la calidad en los procesos clave.

La evaluación de la imagen en la dirección estratégica de hoteles en Cuba debe comprender cambios desde el análisis interno y externo del hotel donde se incluyan las perspectivas de los clientes y empleados en la determinación del rumbo estratégico del mismo Esto permite trabajar en la conformación de la imagen deseada y en la implementación del enfoque integral al cliente desde la planificación de los servicios y no solo desde la proyección al mercado como se realiza hasta el momento.

El procedimiento durante sus fases, al incluir la evaluación de la imagen en la dirección estratégica, alinea los mensajes con la visión del hotel, posibilita el diseño de estrategias de comunicación que no distorsionen la realidad, donde se implican diferentes actores en el tiempo. Al considerar estos aspectos los expertos determinaron que el procedimiento implementa el modelo teórico que lo sustenta, cumple con los objetivos que se propone, es sistémico e integral, posee valor metodológico, racionalidad, capacidad de mejoramiento; consistencia lógica, carácter participativo, flexibilidad, viabilidad y estructuración. 
El Hotel Brisas Trinidad del Mar tiene una imagen positiva, aunque existen aspectos que inciden de manera desfavorable en su sostenibilidad. Estos incluyen la proyección de mensajes que no reflejan de manera coherente la realidad, aspectos de la identidad que no sirven de soporte a la imagen deseada, y la proyección de estrategias respecto a procesos clave del hotel que no solucionan las debilidades que inciden de manera negativa en la imagen percibida por los turistas.

El procedimiento propuesto es capaz de lograr la mejora de la percepción de la imagen del hotel en los turistas, posee además un impacto positivo en el índice de satisfacción, lo que se valida con la aplicación integral y sistemática del procedimiento en el hotel Brisas Trinidad del Mar y en siete estudios de casos en pequeños y medianos hoteles en Cuba; al compararse mediante el análisis de factibilidad la situación con y sin procedimiento, se puede afirmar que este presenta resultados favorables a su implementación.

\section{Limitaciones del estudio}

A continuación se enumeran las limitaciones del estudio realizado. Limitación 1: la imagen con que llegan los turistas al hotel no se mide en los mercados emisores directamente a través de encuestas, sino que se infiere a partir del análisis de contenido de los principales mensajes promocionales a que los públicos están expuestos. Limitación 2: dentro de la propuesta se establecen las técnicas de investigación necesarias para demostrar la fiabilidad y validez de las escalas en la medición de la imagen primaria en los clientes actuales, pero no las suficientes. Las técnicas que se suponen suficientes conllevan al menos mayor tiempo de aplicación y un nivel superior de dominio de la estadística para los usuarios del procedimiento. La aplicación de dichas técnicas tienen sentido desde el punto de vista científico, pero en la práctica cotidiana van en contra del criterio de racionalidad. Limitación 3: los resultados que corresponden a la imagen general de la cadena consideran la suma holística de las imágenes de los hoteles que la componen, donde no es posible conocer sobre otro componente que no sea el cognitivo y en aspectos predefinidos por un agente externo a la investigación. No obstante, la opción de obtener información primaria directa y completa supone la no rentabilidad de la misma para los propósitos de este procedimiento. La fuente de información identificada para este fin es el sitio web tripadvisor disponible en http://www.tripadvisor.es.

\section{Futuras líneas de investigación}

Generación y validación de escalas para la medición de la imagen en otras tipologías de hoteles no contempladas.

Diseño de modelos de gestión integral de la imagen del destino, cadena y hotel.

Propuesta de instrumentos informatizados para la generalización y validación de dimensiones y atributos para medir la imagen del destino, la cadena y el hotel que permitan encuestar online.

Incorporación a la evaluación de la imagen de hoteles, la visión desde las tendencias en el comportamiento de compra del consumidor digital en el turismo

\section{Bibliografía}

Anholt, S. 2003. Branding places and nations. Londres: Profile Books.

Anholt, S. 2014. Marca nación con autoridad moral: una perspectiva distinta. Temas, 1(77): 4-13.

Arteaga, D. 2011. La Calidad en el servicio como elemento diferenciador de imagen en una empresa de telecomunicaciones que ofrece servicios empresariales de datos en Internet. Tesis de Licenciatura. Ecuador: Departamento de Comunicación, Universidad Técnica Particular de Loja, Quito.

Beerli, A., Martín, J. y Moreno, S. 2008. La concepción sistémica de la imagen del destino: una aproximación desde la imagen del alojamiento. [En línea] Disponible en: www.esade.es/cedit2004/ pdfs/41_Moreno.pdf [Último acceso: 27 mayo 2017].

Boorstin, D. 1962. The image: Or what happened to the American dream. New York: Atheneum.

Capriotti, P. 2013. Planificación estratégica de la imagen corporativa. 4ta Ed. Málaga: Editorial Ariel.

Chu, R. y Choi, T. 2000. An importance-performance analysis of hotel selection factors in the Hong

Kong hotel industry: a comparison of business and leisure travellers. Tourism Management, 21(4), pp. 363-377. 
Costa, J. 2008. El concepto del término imagen. Disponible en: www.rrppnet.com.ar/imagencorporativares.htm.

Cupa, U. 2010. Modelo de dirección estratégica implementando el cuadro de mando integral para la pequña empresa. Andalucía: Universidad Internacional de Andalucía.

Duffus, D. 2015. Evaluación de la imagen en la dirección estratégica de pequeños y medianos hoteles en Cuba. Tesis de doctorado: Departamento de Economía. Universidad Central "Marta Abreu" de Las Villas.Cuba.

Duffus, D. y Pons, R. 2014. Propuesta de procedimiento para medir la imagen de hoteles de la Región Central de Cuba. Revista Retos Turísticos. Disponible en: http://retos.umcc.cu//index.

Echevarría, F. 2010. La imagen pública. Un valor de comunicación. Revista Icono, 2(8): 264-275.

Eric, K. y Mark, F. 2010. The role of image and reputation as intangible resources in Non-Profit Organisations: A Relationship Management Perspective. Hong Kong, Published by Academic Publishing Limited.

Goodrich, J. L. 1977. A new approach to image analysis though multidimensional scaling. Journal of Travel Research, 3(16): 3-7.

Huawen, S. 2015. Critical success factors for leading hotel brands in Asia: a case study of Banyan Tree. International Journal of Marketing Studies, 7(3): 19-26.

Lawshe, C. H. 1975. A quantitative approach to content validity. Personnel Psychology, 28: 563-575.

Manrrique, O. y López, J. 2012. Imagen corporativa bajo la tendencia minimalista para empresas de servicios publicitarios 3037 CA., orientado a un mercado de clientes fijos, Caracas, Venezuela: Tesis en opción al grado de Licenciatura en Administración de Empresas de Diseño.

Martín, R. 2014. ¿Está estancado el sector turístico cubano? El desempeño del turismo en Cuba en una visión de largo plazo, Trinidad, Editorial Samuel Feijóo, UCLV, pp. 1-15.

Martínez, Y., Laria, L., Cueto, Y. y Alfonso, Y. 2012. Procedimiento para la elaboración de la estrategia de comunicación en el hotel Zaza, Sancti Spíritus, Cuba, Sancti Spíritus: Universidad de Sancti Spíritus.

Ministerio de Turismo (MINTUR). 2014. Resolución 186: Política de comunicación promocional para el Sistema del Ministerio de Turismo, La Habana: Ministerio de Turismo de Cuba.

Moreno, S. 2008. La concepción sistémica de la imagen del destino: una aproximación desde la imagen del alojamiento. Disponible en: www.esade.es/cedit2004/pdfs/41_Moreno.

Muñiz, N. 2009. Marketing de ciudades; casos exitosos en el mundo de transformación y promoción urbana. En: J. Munueray R. Ana, edit. Casos de marketing estratégico en las organizaciones. Madrid: ESIC, pp. 40-60.

Oficina Nacional de Estadística e Información (ONEI) 2015. Anuario estadístico de Cuba», La Habana: ONEI.

Perelló, J.L. 2014. Imagen país. Promoción del turismo. Temas, enero-marzo, 77: 43-48.

Pike, S. y Ryan, C. 2004. Destination Positioning Analysis through a Comparison of Cognitive, Affective, and Conative Perceptions. Journal of Travel Research, 42(4): 333-342.

San Martín, H. 2005. Estudio de la imagen de destino turístico y el proceso global de satisfacción: adopción de un enfoque integrador. Santander: Tesis en opción al grado de Doctorado en Administración de Empresas de la Universidad de Cantabria.

Sanz de la Tajada, L. A. 1994. Integración de la identidad y la imagen de la empresa: aplicación conceptual y práctica. [s.l.]: ESIC.

Soares,J.R y Godoi, C.K 2017. A metodologia da análise sociológica do discurso em estudos turísticos: o processo de transformação da imagem turística e sua relação com a lealdade. Revista Pasos, 15 (1): 245-260. https://doi.org/10.25145/j.pasos.2017.15.015

Tanda, J. 2011. Fijación de la identidad urbana deseada en la gestión de ciudades: caso Ciudad de Matanzas. Tesis de doctorado: Departamento de Economía y turismo, Universidad de Matanzas "Camilo Cienfuegos".Cuba.

\section{Notes}

1 En esta investigación se entiende la imagen primaria como la percepción cognitiva, afectiva con implicaciones conativas que el visitante experimenta durante su estancia en el hotel. Esta definición corresponde a la tipología de la imagen según la experiencia previa definida por Goodrich (1977); Beerli et al., (2008). Y coincide con la imagen constituida definida por Soares y Godoi (2017). 
2 Se entiende como la representación cognitiva y afectiva formada en el turista a partir de fuentes externas al hotel previa a su estancia en el mismo. Esta definición corresponde a la tipología de la imagen según a experiencia previa, definida por Goodrich, 1977; Beerli et al., 2008.

3 Existen múltiples modas; se muestra el menor valor.

4 El software fue escrito en Java como aplicación de desktop, muestra una pantalla inicial que recoge características del hotel según su categoría, modalidad y tamaño y en consecuencia ofrece tipologías de cuestionarios ajustados. Luego de aplicar los cuestionarios, se introducen los datos y el SEIH vs 1 proporciona como resultado principal un listado de fortalezas, debilidades, amenazas y oportunidades respecto a la identidad y la imagen del hotel. Al usuario se le ofrecen facilidades como atributos de medida válidos y fiables. Además no es necesario dominar la estadística para interpretar los resultados.

5 Estos no son los únicos aspectos a considerar en la formulación de los objetivos y las estrategias, esta propuesta es solo una contribución al subsistema de mercadotecnia, administración y producción para un abordaje integral y coherente en el logro de la imagen deseada. 